\title{
Esophageal thermal injury in catheter ablation of atrial fibrillation
}

\author{
Takashi Kaneshiro and Yasuchika Takeishi \\ Department of Cardiovascular Medicine/Arrhythmia and Cardiac Pacing, Fukushima Medical Univer- \\ sity
}

(Received August 25, 2021, accepted September 27, 2021)

\begin{abstract}
Pulmonary vein isolation is an established method for the catheter ablation of atrial fibrillation. Esophageal thermal injuries, such as esophageal erosion, ulceration and periesophageal nerve injury leading to gastric hypomotility, are important complications associated with pulmonary vein isolation. In this review article, we describe the mechanisms, characteristics and the predictors of esophageal thermal injury associated with pulmonary vein isolation.
\end{abstract}

Key words : atrial fibrillation, catheter ablation, esophageal thermal injury, esophageal mucosal lesion, periesophageal nerve injury

\section{Introduction}

Since the identification of the pulmonary vein $(\mathrm{PV})$ as the trigger for atrial fibrillation (AF), $\mathrm{PV}$ isolation (PVI) has been a common method for the catheter ablation of AF, especially in patients with paroxysmal $\mathrm{AF}^{1,2)}$. Pulmonary vein isolation has been shown to be effective in preventing AF by the creation of a bidirectional conduction block between the left atrium (LA) and $\mathrm{PV}^{3)}$. In contrast, a systematic approach to reduce esophageal thermal injury (ETI) in the context of PVI, such as an esophageal mucosal lesion and periesophageal nerve injury leading to gastric hypomotility, has yet to be established. This can have serious consequences as esophageal mucosal injury has the potential to advance to a left atrial-esophageal fistula, which is a fatal complication associated with $\mathrm{PVI}^{4,5}$. In this review article, we describe the mechanisms, characteristics and the predictors of ETI associated PVI.

\section{Catheter ablation of AF}

Pulmonary vein isolation is at the core of $\mathrm{AF}$ ablation techniques. The aim of PVI is to create a bidirectional block between the LA and each PV, as most abnormal triggering activity for AF could origi- nate from either PV. Radiofrequency-based PVI has been shown to be superior to pharmacological treatments using antiarrhythmic drugs in patients with paroxysmal $\mathrm{AF}^{3,6)}$. Since catheter ablation for AF has been traditionally performed using radiofrequency energy, multiple ablation lesions around the ostium of the PV are needed (Figure 1). Therefore, the risk of various complications associated with $\mathrm{AF}$ ablation has not been overcome sufficiently.

\section{Advancement of catheter ablation equipment}

In radiofrequency-based catheter ablation, the development of ablation equipment, such as advancements in 3-dimensional (3D) navigation systems and contact force $(\mathrm{CF})$ monitoring catheters, has led to AF ablation becoming much safer and more effective ${ }^{7,8)}$. Moreover, the use of lesion quality markers has become more widely accepted for $\mathrm{AF}$ ablations $\mathrm{s}^{9,10)}$. Recently, high-power shortduration (HP-SD) ablation has been introduced in $\mathrm{AF}$ ablation. High-power short-duration ablation can achieve uniform, transmural lesions during the resistive heating phase, and reduce collateral tissue damage by shortening the conductive heating phase $^{11,12)}$. High-power short-duration ablation

Corresponding author : Takashi Kaneshiro, MD. E-mail : tk2435@fmu.ac.jp

(C)2021 The Fukushima Society of Medical Science. This article is licensed under a Creative Commons [Attribution-NonCommercial-ShareAlike 4.0 International] license.

https://creativecommons.org/licenses/by-nc-sa/4.0/ 


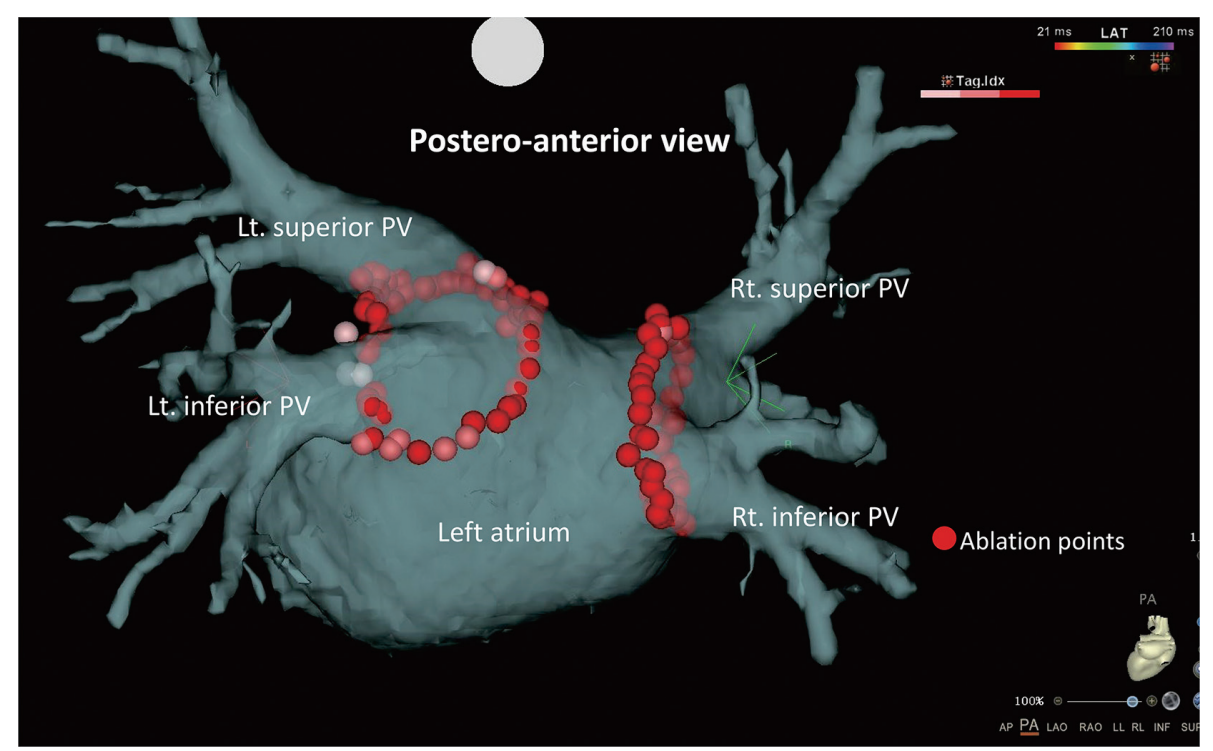

Fig. 1. Schematic image of 3-dimenonal navigation system during radiofrequency pulmonary vein isolation. Postero-anterior view of 3-dimenonal navigation system during radiofrequency pulmonary vein isolation. The ostium of each PV was circumferentially ablated using radiofrequency ablation catheter.

$\mathrm{PV}$, pulmonary vein.

combined with a lesion quality marker might have strong potential in improving safety and efficacy for AF ablation. Recently, PVI using cryoballoon (CB) has been widely accepted as a treatment for $\mathrm{AF}$ (Figure 2). Pulmonary vein isolation using secondgeneration CB (Arctic Front Advance, Medtronic, Inc., Minneapolis, MN, USA) has a high cooling effect that can achieve durable lesions of the PV antrum and better clinical outcomes ${ }^{13,14}$. With the development of such ablation equipment, the incidence of cardiac tamponade, which had previously been recognized as the most frequent complication of AF ablation, has decreased. On the other hand, the mechanisms underlying ETI have not been established and a preventative strategy has yet to be described.

\section{Esophageal thermal injury in AF ablation}

Esophageal thermal injuries, such as esophageal erosion, ulceration and periesophageal nerve injury leading to gastric hypomotility, are important in the catheter ablation of AF. Esophageal thermal injury has the potential to advance into a left atrialesophagus fistula, which is a fatal complication associated with $\mathrm{PVI}^{4,5)}$. Various factors, such as LA en-
Left atriography: antero-posterior view

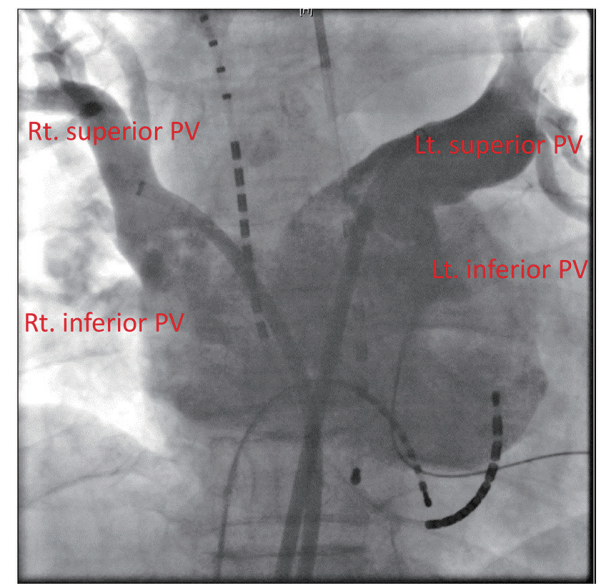

Cryoballoon ablation for It. superior PV

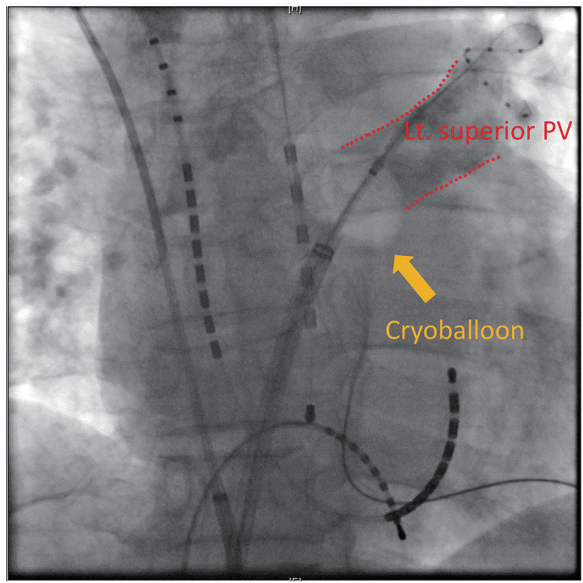

Fig. 2. X-ray images during cryoballoon pulmonary vein isolation. Cryoballoon catheter was positioned and occluded the ostium of left superior PV. PV, pulmonary vein. 
largement, distance between the LA and esophagus, further ablation in addition to PVI and body mass index (BMI), have been reported to be associated with ETI in radiofrequency catheter ablation ${ }^{15,16)}$. However, predictors of ETI concerning the anatomical proximity of the structures surrounding the esophagus have not been fully evaluated in radiofrequency PVI and CB-PVI.

\section{Anatomical predisposing factors in ETI}

Our work has focused on this gap in the literature, where we have explored the anatomical proximity of the structures surrounding the esophagus and subsequent ETI after PVI using radiofrequency and CB-PVI. Based on electrocardiogram-gated computed tomography, we analyzed three anatomical factors to predict ETI after PVI. First, we analyzed the angle of the LA posterior wall to the descending aorta (LA-Ao angle) as shown in Figure 3A. We calculated LA-Ao angle in the left lateral view shown in the $3 \mathrm{D}$-reconstructed CT imaging. We adjusted the projection angle to see the LA posterior wall at a tangent. Then, we calculated LA-Ao angle as the angle between the LA posterior wall and the anterior surface of descending Ao. The surface of the LA posterior wall bends forward and touches the anterior aspect of the esophagus. If the LA-Ao angle is small, the contact area of the LA posterior wall and esophagus might be larger. Second, we measured the branching angle of the left inferior PV to the coronal plane (LIPV angle) as demonstrated in Figure 3B. We calculated the LIPV angle as the angle between the line which runs longitudinally in the center of LIPV and coronal plane from horizontal CT imaging, in which the ostium of LIPV is rendered. As mentioned above, the anterior aspect of the esophagus touches the LA posterior wall in the lower portion. The LIPV branches backwards, therefore if the LIPV angle is larger, the ostium of LIPV becomes closer to esophagus. Third, the distance between the descending aorta and LA posterior wall enclosing the esophagus (LA-Ao distance) was calculated as in Figure 3C. We evaluated the LA-Ao distance through horizontal CT imaging, in which the ostium of LIPV is rendered. We calculated the LA-Ao distance as the distance between the LA posterior wall and the anterior surface of the descending Ao, which encloses the esophagus. The distance between the LA posterior wall and the descending Ao is the shortest distance at the level of LIPV ostium, because the LA posterior wall bends forward and is closest to the anterior surface of the descending Ao. Due to the lack of fixation in the posterior mediastinum the esophagus is a mobile or$\operatorname{gan}^{17)}$, therefore the distance from the LA posterior wall to the esophagus can vary and is an unreliable parameter to use in proximity evaluation between the esophagus and these structures. The esophagus is located between the LA posterior wall and the descending Ao. Therefore, if the LA-Ao distance was smaller, the proximity between LA posterior wall and esophagus might become closer through a sandwiching mechanism due to catheter contact force.

We performed an analysis of 110 patients who
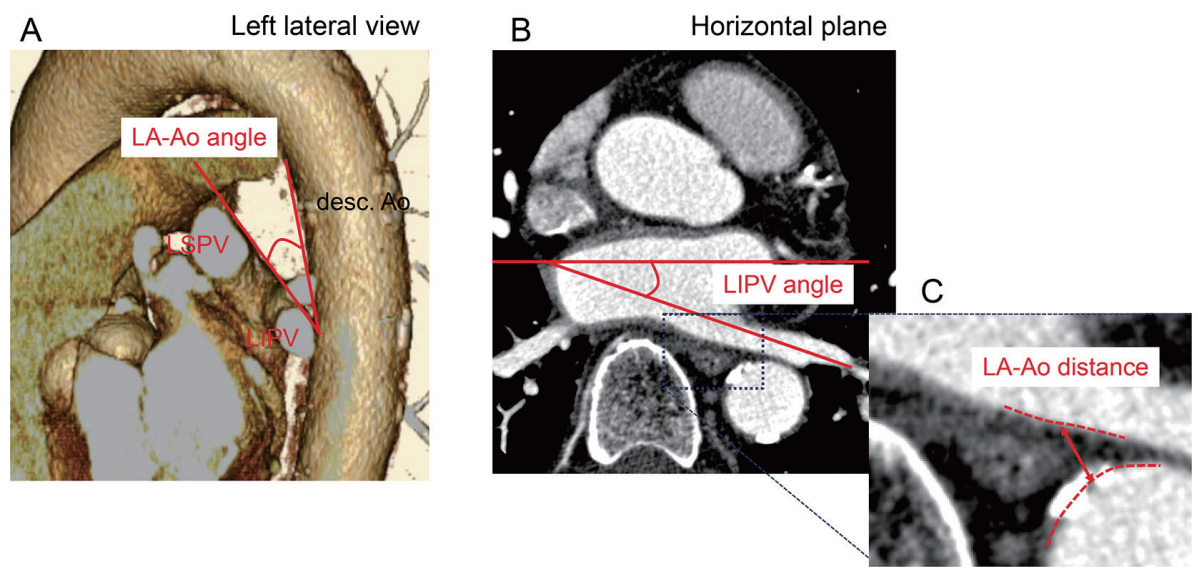

Fig. 3. The parameters used to evaluate proximities of the esophagus to its surrounding structures on the computed tomography data.

A : LA-Ao angle : the angle of the LA posterior wall to the descending Ao.

$\mathrm{B}$ : LIPV angle : the branching angle of the LIPV to the coronal plane.

$\mathrm{C}:$ LA-Ao distance : the distance between the descending Ao and LA posterior wall.

Ao, aorta ; LA, left atrium ; LIPV, left inferior pulmonary vein ; LSPV, left superior pulmonary vein.

Modified from the figure in the article by Kaneshiro T, et al. ${ }^{18)}$ 
underwent radiofrequency PVI (mean age $62 \pm 9$ years, 18 female), where 21 out of 110 patients (19\%) had ETIs, including esophageal erosion in five patients and gastric hypomotility in 16 patients (Figure 4$)^{18)}$. No patients had ETI-associated symptoms. When comparing the groups with and without ETI, the LIPV angle was significantly larger in the ETI (+) group compared to that in the ETI (-) group (33.4 \pm 14.5 vs. $21.6 \pm 10.6$ degrees, $p<$ 0.001). Additionally, the LA-Ao distance was significantly smaller in the ETI $(+)$ group compared to that of the ETI ( -$)$ group $(4.5 \pm 1.3$ vs. $6.3 \pm 2.5$ $\mathrm{mm}, p=0.003)$. However, the LA-Ao angle was not significantly different between the groups with and without ETI $(25.9 \pm 10.4$ vs. $29.1 \pm 10.1$ degrees, $p=0.230$ ). Based on these results, we concluded that the anatomical structures surrounding the esophagus, especially the LIPV angle and LA-Ao distance were associated with the development of
ETI.

We also analyzed 110 consecutive patients (mean age $64 \pm 9$ years, 30 female), who had undergone their first CB-PVI for drug refractory AF. Nineteen of the 110 patients (17\%) had ETIs, including esophageal erosion in two patients and gastric hypomotility in 17 patients (Figure 5$)^{19}$. No patients had ETI-associated symptoms. When comparing the groups with and without ETI, the LIPV angle in the ETI $(+)$ group was significantly larger $(24.5 \pm 11.2$ vs. $20.0 \pm 8.1 \mathrm{~mm}, p=0.047)$ and the LA-Ao distance was significantly smaller $(4.9 \pm 1.3$ vs. $5.8 \pm 1.6 \mathrm{~mm}, p=0.030)$ compared to those in the ETI (-) group. Moreover, the patient age was significantly higher in patients with ETI than those without $(68 \pm 8$ vs. $63 \pm 9 \mathrm{~mm}$, $p=0.026$ ). Aging may lead to compromised periesophageal circulation, which combined with the anatomical proximity of the esophagus and surround-
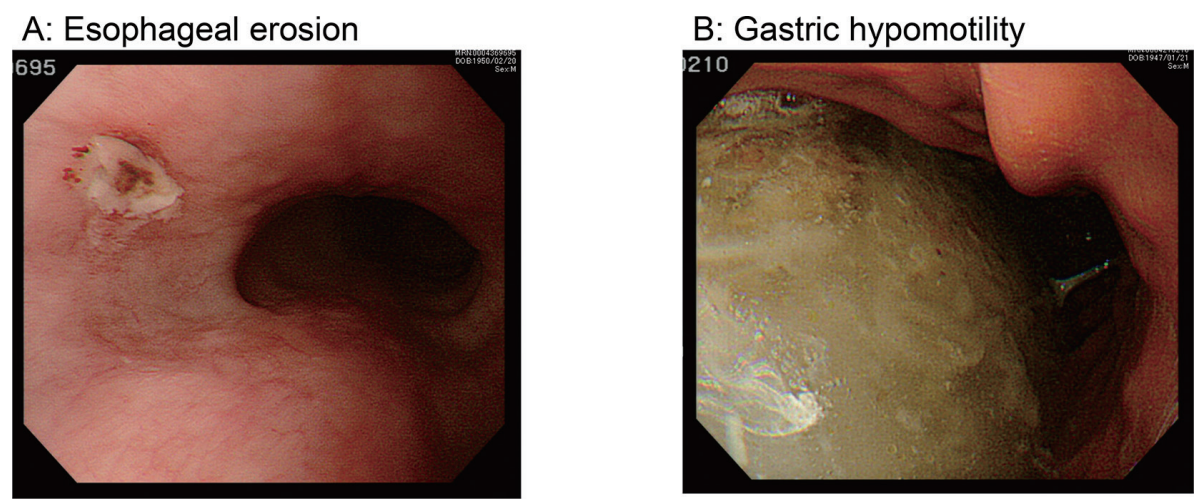

Fig. 4. Representative case of esophageal thermal injury in radiofrequency pulmonary vein isolation.

The panel A shows esophageal erosion and the panel B shows gastric hypomotility, which were revealed by esophagogastroduodenoscopy performed after PVI.

PVI, pulmonary vein isolation.

Modified from the figure in the article by Kaneshiro T, et al. ${ }^{18)}$
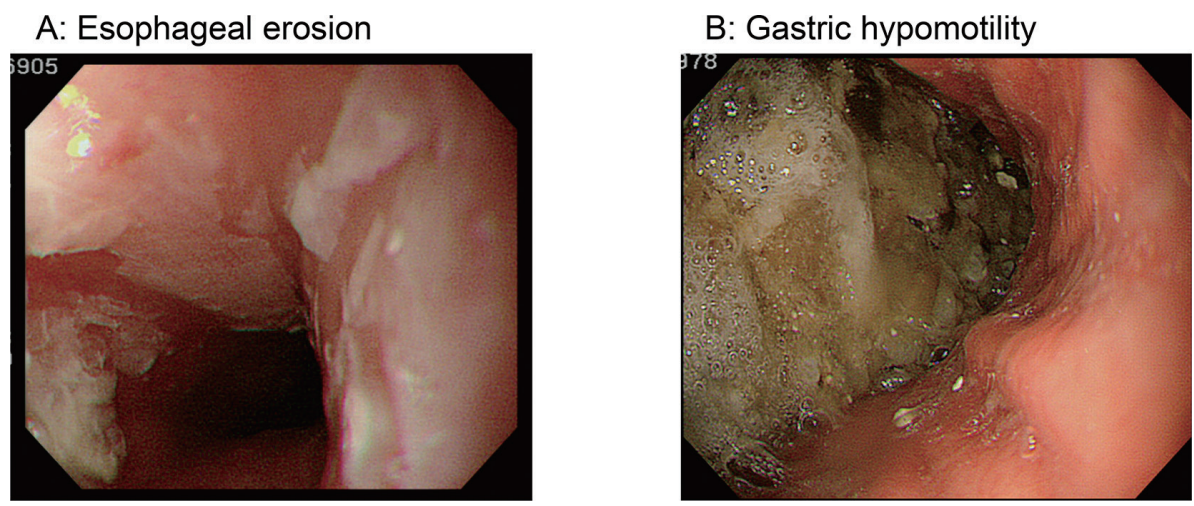

Fig. 5. Representative cases of esophageal thermal injury in cryoballoon pulmonary vein isolation.

The panel A shows esophageal erosion and the panel B shows gastric hypomotility, which were revealed by esophagogastroduodenoscopy performed after PVI.

PVI, pulmonary vein isolation.

Modified from the figure in the article by Matsumoto Y, et al. ${ }^{19)}$ 
ing structures are important contributing factors in the prevalence of ETI in CB-PVI. All patients undergoing radiofrequency PVI who developed esophageal lesions showed spot lesions on the anterior wall of the esophagus (Figure 4A), which touches the left atrial posterior wall. However, most esophageal lesions showed a linear, longitudinal form in patients who had CB-PVI (Figure 5A). These differences in the form of the esophageal mucosal lesions might depend on the energy sources involved ${ }^{20)}$.

These results suggest that spatial positioning in relation to adjacent structures surrounding the esophagus are important in the prevalence of ETI regardless of the power source.

\section{ETI in HP-SD ablation}

Recently, a high-power short-duration (HP-SD) setting has been introduced in AF ablation ${ }^{11,12)}$. Ablation using this setting could achieve uniform, transmural lesions during the resistive heating phase and reduce collateral tissue damage by shortening the conductive heating phase. However, the incidence and characteristics of ETI such as esophageal mucosal injury and periesophageal nerve injury leading to gastric hypomotility when using the HPSD setting remain unknown. We therefore analyzed 271 consecutive patients $(62 \pm 10$ years, 56 female) who underwent PVI by radiofrequency catheter ablation. In 101 patients, a HP-SD setting at 45-50 W was used (HP-SD group). In the remaining 170 patients who underwent treatment before the introduction of the HP-SD setting, a conventional power setting of 20-30 W was used (conventional group). Seventy-five out of 271 patients (28\%) had ETIs, including esophageal lesions in 20 patients $(7 \%)$ and gastric hypomotility in 61 patients $(23 \%)^{21)}$. The prevalence of ETI was significantly higher in the HP-SD group than in the conventional group (37\% vs. $22 \%, p=0.011$ ). Although the prevalence of esophageal lesions did not differ between the groups (7\% in the HP-SD group vs. $8 \%$ in the conventional group), the complication of gastric hypomotility was significantly higher in the HP-SD group than the conventional group (33\% vs. 16\%, $p=0.002)$. Moreover, all esophageal lesions were erythematous in the HP-SD group, whereas the esophageal lesions showed ulceration in the conventional group (Figure 6). When comparing patients with and without ETI, the HP-SD setting was used ( $49 \%$ vs. $33 \%, p=0.011$ ) more frequently in patients with ETI than in those without; furthermore the LIPV angle was significantly larger $(29.4 \pm 11.5$ degrees vs. $19.0 \pm 9.6$ degrees, $p<0.001$ ), and the LA-Ao distance was significantly smaller $(5.2 \mathrm{~mm} \pm$ 1.2 vs. $6.7 \pm 2.0 \mathrm{~mm}, p<0.001$ ) in the patients with ETI compared to those without. These results suggest that the lesion characteristics made by RF energy power may influence the development and characteristics of ETI, impacting on ETI prevalence.

\section{For the prevention of ETI}

Due to the results of our study series, we have

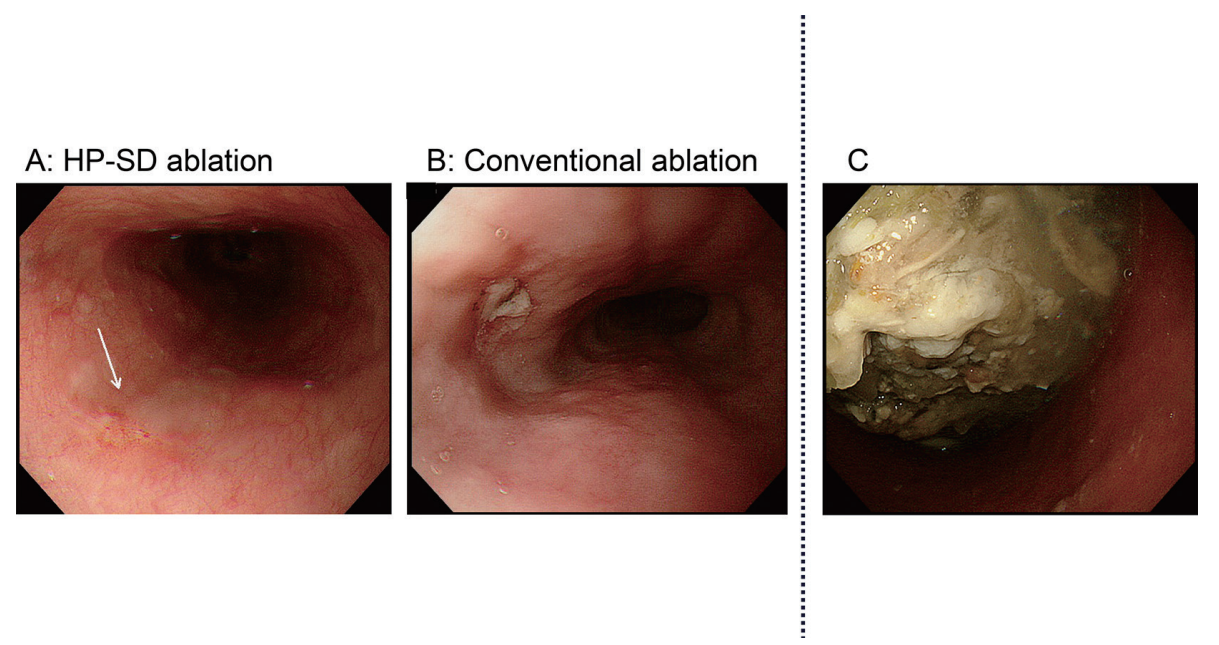

Fig. 6. Representative cases of esophageal thermal injury in high-power short-duration and conventional ablations. The panel A shows a representative figure of esophageal erythema (arrow) in the high power-short duration setting. The panel B shows a representative figure of esophageal ulcer in the conventional setting. The panel C shows a representative figure of gastric hypomotility in the high-power short-duration setting. Modified from the figure in the article by Kaneshiro T, et al. ${ }^{21)}$ 
revealed that the anatomical proximity of the esophagus and the fixed structures surrounding the esophagus has a large impact on the prevalence of ETI after AF ablation. Moreover, the HP-SD ablation, an advanced radiofrequency ablation setting, has strong potential in avoiding a deeper thermal injury to the esophageal mucosal layer. Based on the mechanisms and contributing factors of ETI development outlined in this article, we recommend using a HP-SD ablation setting to minimize ETI.

\section{Acknowledgements}

The authors would like to acknowledge Professor Yukio Maruyama for his direction, support and valuable advice. The authors also would like to thank all those who contributed to these studies.

\section{References}

1. Haïssaguerre M, Jaïs P, Shah DC, et al. Spontaneous initiation of atrial fibrillation by ectopic beats originating in the pulmonary veins. N Engl J Med, 339 : 659-666, 1998.

2. Calkins H, Reynolds MR, Spector P, et al. Treatment of atrial fibrillation with antiarrhythmic drugs or radiofrequency ablation : two systematic literature reviews and meta-analyses. Circ Arrhythm Electrophysiol, 2 : 349-361, 2009.

3. Wilber DJ, Pappone C, Neuzil P, et al. ; ThermoCool AF Trial Investigators. Comparison of antiarrhythmic drug therapy and radiofrequency catheter ablation in patients with paroxysmal atrial fibrillation : a randomized controlled trial. JAMA, 303 : 333-340, 2010.

4. Pappone C, Oral H, Santinelli V, et al. Atrioesophageal fistula as a complication of percutaneous transcatheter ablation of atrial fibrillation. Circulation, 109 : 2724-2726, 2004.

5. Dagres N, Kottkamp H, Piorkowski C, et al. Rapid detection and successful treatment of esophageal perforation after radiofrequency ablation of atrial fibrillation: lessons from five cases. J Cardiovasc Electrophysiol, 17 : 1213-1215, 2006.

6. Calkins H, Kuck KH, Cappato R, et al. 2012 HRS/ EHRA/ECAS Expert Consensus Statement on Catheter and Surgical Ablation of Atrial Fibrillation : recommendations for patient selection, procedural techniques, patient management and follow-up, definitions, endpoints, and research trial design. Europace, 14 : 528-606, 2012.

7. Kautzner J, Neuzil P, Lambert H, et al. EFFICAS II : optimization of catheter contact force improves outcome of pulmonary vein isolation for paroxysmal atrial fibrillation. Europace, 17 : 1229-1235, 2015.

8. Chikata A, Kato T, Sakagami S, et al. Optimal force-time integral for pulmonary vein isolation according to anatomical wall thickness under the ablation line. J Am Heart Assoc, doi : 10.1161/ JAHA.115.003155, 2016.

9. Das M, Loveday JJ, Wynn GJ, et al. Ablation index, a novel marker of ablation lesion quality : prediction of pulmonary vein reconnection at repeat electrophysiology study and regional differences in target values. Europace, 19: 775-783, 2017.

10. Hussein A, Das M, Chaturvedi V, et al. Prospective use of Ablation Index targets improves clinical outcomes following ablation for atrial fibrillation. J Cardiovasc Electrophysiol, 28 : 1037 1047, 2017.

11. Castrejón-Castrejón S, Martínez Cossiani M, Ortega Molina M, et al. Feasibility and safety of pulmonary vein isolation by high-power short-duration radiofrequency application : short-term results of the POWER-FAST PILOT study. J Interv Card Electrophysiol, 57 : 57-65, 2020.

12. Winkle RA, Mohanty S, Patrawala RA, et al. Low complication rates using high power (45-50 W) for short duration for atrial fibrillation ablations. Heart Rhythm, 16 : 165-169, 2019.

13. Ciconte G, de Asmundis C, Sieira J, et al. Single 3-minute freeze for second-generation cryoballoon ablation : one-year follow-up after pulmonary vein isolation. Heart Rhythm, 12 : 673-680, 2015.

14. Su W, Kowal R, Kowalski M, et al. Best practice guide for cryoballoon ablation in atrial fibrillation : the complication experience of more than 3000 procedures. Heart Rhythm, 12 : 1658-166, 2015.

15. Martinek M, Meyer C, Hassanein S, et al. Identification of a high-risk population for esophageal injury during radiofrequency catheter ablation of atrial fibrillation: procedural and anatomical considerations. Heart Rhythm, 7 : 1224-1230, 2010.

16. Yamasaki H, Tada H, Sekiguchi Y, et al. Prevalence and characteristics of asymptomatic excessive transmural injury after radiofrequency catheter ablation of atrial fibrillation. Heart Rhythm, 8: 826-832, 2010.

17. Good E, Oral H, Lemola K, et al. Movement of the esophagus during left atrial catheter ablation for atrial fibrillation. J Am Coll Cardiol, 46 : 2107-2110, 2005.

18. Kaneshiro T, Matsumoto Y, Nodera M, et al. Anatomical predisposing factors of transmural thermal injury after pulmonary vein isolation. Europace, 20 : 1122-1128, 2018.

19. Matsumoto Y, Kaneshiro T, Hijioka N, et al. Predicting factors of transmural thermal injury after 
cryoballoon pulmonary vein isolation. J Interv Card Electrophysiol, 54 : 101-108, 2019.

20. Kaneshiro T, Matsumoto Y, Hijioka N, et al. Distinct forms of esophageal lesions after radiofrequency and cryoballoon pulmonary vein isolation. JACC Clin Electrophysiol, 4 : 1642-1643,
2018.

21. Kaneshiro T, Kamioka M, Hijioka N, et al. Characteristics of esophageal injury in ablation of atrial fibrillation using a high-power short-duration setting. Circ Arrhythm Electrophysiol, 13 : e008602, 2020. 